\title{
Tentative evidence of spatially extended GeV emission from SS433/W50
}

\author{
Xiao-Na Sun ${ }^{1,2}$, Rui-Zhi Yang ${ }^{3}$, Bing Liu ${ }^{1,3}$, Shao-Qiang Xi ${ }^{1,2}$, and Xiang-Yu Wang ${ }^{1,2}$ \\ ${ }^{1}$ School of Astronomy and Space Science, Nanjing University, Nanjing 210093, PR China \\ e-mail: sxn@nju.edu.cn \\ ${ }^{2}$ Key laboratory of Modern Astronomy and Astrophysics, Nanjing University, Ministry of Education, Nanjing 210093, PR China \\ ${ }^{3}$ Max-Planck-Institut für Kernphysik, Saupfercheckweg 1, 69117 Heidelberg, Germany
}

Received 4 April 2019 / Accepted 15 May 2019

\begin{abstract}
We have analyzed ten years of the Fermi Large Area Telescope data toward the SS433/W50 region. With the latest source catalog and diffuse background models, the $\gamma$-ray excess from SS433/W50 is detected with a significance of $\sim 6 \sigma$ in the photon energy range of $500 \mathrm{MeV}-10 \mathrm{GeV}$. Our analysis indicates that an extended flat disk morphology is preferred over a point-source description, suggesting that the $\mathrm{GeV}$ emission region is much larger than that of the $\mathrm{TeV}$ emission detected by the High Altitude Water Cherenkov (HAWC) telescope. The size of the GeV emission is instead consistent with the extent of the radio nebula W50, a supernova remnant being distorted by the jets, so we suggest that the $\mathrm{GeV}$ emission may originate from this supernova remnant. The spectral result of the $\mathrm{GeV}$ emission is also consistent with a supernova remnant origin. Furthermore, we derive the GeV flux upper limits on the $\mathrm{TeV}$ emission region, which put moderate constraints on the leptonic models to explain the multiwavelength data.
\end{abstract}

Key words. gamma rays: general - supernovae: individual: SS433/W50

\section{Introduction}

The Galactic X-ray binary system SS433 contains a compact object (either a black hole or a neutron star) accreting matter from a companion star (Gies et al. 2002; Eikenberry et al. 2001). It is the prototype of microquasars and has been studied intensively due to its exceptional brightness and power (for a review, see e.g., Mirabel \& Rodríguez 1999). It is located close to the geometric center of the radio nebula W50, which is most likely a supernova remnant (SNR). Several X-ray hotspots located west of the central binary (w1 and w2) and east (e1, e2, e3) are observed (Safi-Harb \& Ögelman 1997). These regions are bright at X-rays due to the interaction between the jets and the ambient medium (Safi-Harb \& Ögelman 1997; Safi-Harb \& Petre 1999; Brinkmann et al. 2007). Two bright radio ears to the east and west of the nebula are generally believed to be punched out by two oppositely directed relativistic jets (Abell \& Margon 1979; Fabian \& Rees 1979; Margon et al. 1979; Milgrom 1979; Dubner et al. 1998) moving outward from SS433 (Margon 1984; Elston \& Baum 1987; Goodall et al. 2011a,b; Farnes et al. 2017). The entire radio morphology of the SS433/W50 system is thus seen as an approximately elliptical shell that is $2^{\circ} \times 1^{\circ}$ in projection on the sky (Geldzahler et al. 1980). S433/W50 lies at a distance of $5.5 \pm 0.2 \mathrm{kpc}$ (Blundell \& Bowler 2004; Lockman et al. 2007), implying a physical size of $180 \mathrm{pc} \times 80 \mathrm{pc}$ (Goodall et al. 2011a).

Bordas et al. (2015) report for the first time the $\mathrm{GeV}$ emission that is tentatively associated with SS433/W50 using five years of Fermi Large Area Telescope (Fermi-LAT) data. Recently, the High Altitude Water Cherenkov (HAWC) telescope detected very-high-energy (VHE) emission $(\sim 20 \mathrm{TeV})$ around two $\mathrm{X}$-ray hotspot regions, e1 and $\mathrm{w} 1$, with a significance of $5.4 \sigma$ (Abeysekara et al. 2018). The spatial extension analysis of the two hotspots yields upper limits on the angular size of the emission regions being $0.25^{\circ}$ for the east hotspot and $0.35^{\circ}$ for the west hotspot at $90 \%$ confidence (Abeysekara et al. 2018). The spectral results indicate that the broadband spectral energy distribution (SED) at the e1 region can be naturally explained within a pure leptonic model, in which the same population of relativistic electrons with energies up to hundreds of $\mathrm{TeV}$ produce the radio to $\mathrm{X}$-ray emission through synchrotron radiation and up-scatter photons from the cosmic microwave background (CMB) to the TeV $\gamma$-rays. Xing et al. (2019) analyze ten years of Fermi-LAT data using the preliminary LAT 8-yr point source list (FL8Y) ${ }^{1}$ and finds excess $\mathrm{GeV}$ emission around the western $\mathrm{X}$-ray hotspot $\mathrm{w} 1$ region. However, we note that the point source FL8Y J1913.3+0515, which is consistent with the position of W50 (SNR G039.7-02.0), has been included in their background model, leading to a reduction of the excess $\gamma$-ray emission from the north-east region. Rasul et al. (2019) focus on the temporal behavior of $\mathrm{GeV}$ emission and find tentative evidence for periodicity at the precession period. They analyze nine years of Fermi-LAT data using the Fermi-LAT four-year catalog (3FGL, Acero et al. 2015) and the foreground models gll_iem_v06.fits and $i s O_{-} P 8 R 3 \_S O U R C E \_V 2 . t x t^{2}$.

Different from the previous works, in this paper we use the most recent Fermi-LAT products to reanalyze the SS433/W50 region. We focus on the spatial extension of the $\mathrm{GeV}$ emission using multiple spatial templates. In Sect. 2 we perform a detailed spatial analysis on the Fermi-LAT data toward SS433. In Sect. 3, we present the spectrum and discuss the radiation mechanism. Section 4 is the discussion and conclusion.

\footnotetext{
https://fermi.gsfc.nasa.gov/ssc/data/access/lat/ $\mathrm{fl} 8 \mathrm{y} /$

2 https://fermi.gsfc.nasa.gov/ssc/data/access/lat/ BackgroundModels.html
} 


\section{Spatial analysis of the Fermi-LAT data}

We have analyzed the Fermi-LAT Pass 8 database toward the SS433/W50 system from August 4, 2008 (MET 239557417) until December 18, 2018 (MET 566826221). We selected both the front and back converted photons at energies from $100 \mathrm{MeV}$ to $10 \mathrm{GeV}$. A $14^{\circ} \times 14^{\circ}$ square region centered at the nominal position of SS433 is considered as the region of interest (ROI). We use the "source" event class, recommended for individual source analysis, and the recommended expression (DATA_QUAL $>0) \& \&($ LAT_CONFIG = $=1)$ to exclude time periods when some spacecraft event affected the data quality. To reduce the background contamination from the Earth's albedo, only the events with zenith angles less than $90^{\circ}$ are included for the analysis. The data have been processed through the current Fermitools from conda distribution ${ }^{3}$ together with the latest version of the instrument response functions (IRFs) P8R3_SOURCE_V2. We used the python module that implements a maximum likelihood optimization technique for a standard binned analysis ${ }^{4}$. For the complex structures at low latitude, it is more reasonable to re-optimize for test statistic (TS) fits through setting the keyword tsmin = true.

In our background model, we use Fermi-LAT eight-year catalog (4FGL, The Fermi-LAT Collaboration 2019) by running the make4FGLxml script ${ }^{5}$ within the ROI enlarged by $5^{\circ}$. We leave the normalizations and spectral indices free for all sources within six degrees away from SS433. For the foreground components, we use the latest Galactic diffuse model gll_iem_v07.fits and isotropic emission model iso_P8R3_SOURCE_V2_v1.txt ${ }^{6}$ with their normalization parameters free.

Generally, high energy maps with higher angular resolution are more suitable for the spatial analysis, but low statistics in the higher energy range may prevent any improvement. Therefore, to balance the effects of these two factors, we select the data in the energy range of $500 \mathrm{MeV}-10 \mathrm{GeV}$ for the analysis. We use the gttsmap tool to evaluate a $2.5^{\circ} \times 2.5^{\circ}$ residual TS map by removing the contribution from all the known sources in our background model defined above. The TS value for each pixel is defined as TS $=-2\left(\ln L_{0}-\ln L_{1}\right)$, where $L_{0}$ is the maximum-likelihood value for null hypothesis and $L_{1}$ is the maximum likelihood with the additional source under consideration. The spectral type of all the added sources for the likelihood ratio test in this section are assumed to be a simple power law. As shown in Fig. 1, a strong $\gamma$-ray excess near SS433's nominal position is apparent after the fitting and subtraction of $\gamma$-rays from the background sources by performing the TS analysis.

At the eastern interaction region, there is almost no $\mathrm{GeV}$ emission, which is roughly consistent with the results reported by Bordas et al. (2015), while TeV excess has been detected by HAWC's observation in this region. Abeysekara et al. (2018) have argued that the $\mathrm{TeV}$ emission originates from the jet termination shock, thus the above difference indicates that the $\mathrm{GeV}$ emission likely originates from the other regions, for example, one of the possibilities is the SNR W50 itself.

\footnotetext{
3 https://github.com/fermi-lat/Fermitools-conda/

4 https://fermi.gsfc.nasa.gov/ssc/data/analysis/ scitools/python_tutorial.html

5 https://fermi.gsfc.nasa.gov/ssc/data/analysis/user/ make4FGLxml . py

6 https://fermi.gsfc.nasa.gov/ssc/data/access/lat/

BackgroundModels.html
}

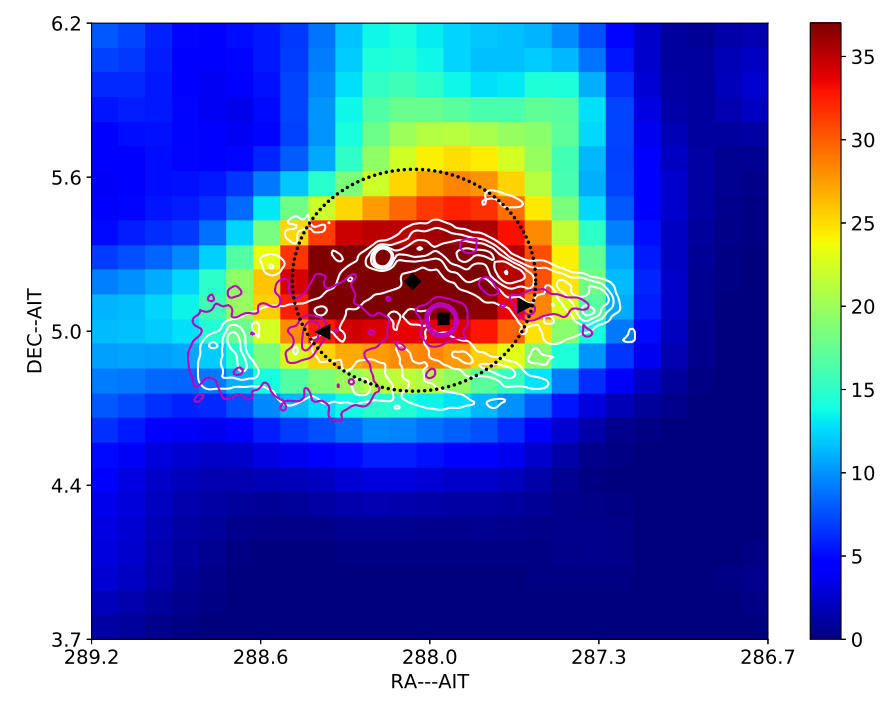

Fig. 1. TS residual map above $500 \mathrm{MeV}$ in the $2.5^{\circ} \times 2.5^{\circ}$ region around SS433/W50 system, with pixel size corresponding to $0.1^{\circ} \times 0.1^{\circ}$. The square shows the nominal position of SS433, and the diamond indicates the best-fit position. The left and right triangles (e1 and w1) mark the fixed positions for the fitting of the VHE excesses detected by HAWC. The contours in white show the main features of the studied region using the radio observation, smoothed with a Gaussian kernel of $0.1^{\circ}$. Contours start at $10 \mathrm{mJy}^{-1}$ beam $^{-1}$ and increase in steps of $40 \mathrm{mJy} \mathrm{beam}^{-1}$. The magenta contours show the X-ray emission, smoothed with a Gaussian filter of $0.17^{\circ}$. The dashed circle with a radius of $0.45^{\circ}$ shows the size of the assumed uniform disk used for spatial analysis.

\subsection{Single point-like source model}

We added a point-like source model encompassing SS433's nominal position into our background model, and optimized the localization using the gtfindsrc tool. The derived best-fit position of the excess above $500 \mathrm{MeV}$ is $\left[\mathrm{RA}=288.02^{\circ}, \mathrm{Dec}=5.18^{\circ}\right]$ (the "diamond" in Fig. 1), with $1 \sigma$ and $2 \sigma$ error radii of $0.4^{\circ}$ and $0.6^{\circ}$, and $0.2^{\circ}$ away from the nominal position. Moreover, SS433's nominal position, e1, and w1 lie within $2 \sigma$ positional errors of the best-fit position. As shown in Table 1, under the assumption of single point-like source model, the significance of the excess $\gamma$-ray emission is $\mathrm{TS}=26(\sim 5 \sigma)$ and the best-fit photon index is $3.31 \pm 0.02$.

In the following, to investigate the morphology and extension of the $\gamma$-ray emission, we consider several spatial templates as the alternative hypothesis, and the above single point-like source model as the null hypothesis. Then we compare the overall maximum likelihood of the alternative hypothesis $(L)$ with that of the null hypothesis $\left(L_{0}\right)$, and define the significance of the alternative hypothesis model $-2\left(\ln L_{0}-\ln L\right)$ following the paper by Lande et al. (2012).

\subsection{Two point-like source model}

The e 1 and w1 regions detected by HAWC (Abeysekara et al. 2018) lie within $2 \sigma$ positional errors of the best-fit position, and we cannot yet rule out the possibility that the $\gamma$-ray emission originates from the regions of the excess $\mathrm{TeV}$ emission. To study whether the extended nature of this $\mathrm{GeV}$ emission is caused by a superposition of two separate point-like sources, we calculated the maximum likelihood value for the most possible combinations of two point-like sources, that is, one point-like source at the 1 location and one point-like source at the w1 position (see Fig. 1). Given that $\Delta \mathrm{TS}=3.4(<2 \sigma)$, this model is disfavored 
Table 1. Spatial analysis results at $500 \mathrm{MeV}-10 \mathrm{GeV}$.

\begin{tabular}{lcccc}
\hline \hline Spatial model & $\begin{array}{c}\text { Energy flux } \\
\left(\times 10^{-11} \mathrm{erg} \mathrm{cm}^{-2} \mathrm{~s}^{-1}\right)\end{array}$ & Power-law index & $-2\left(\ln L_{0}-\ln L\right)^{(b)}$ & $\operatorname{DoF}^{(c)}$ \\
\hline $\mathrm{A}^{(a)}$ & $2.80 \pm 0.13$ & $3.31 \pm 0.02$ & 0 & 0 \\
$\mathrm{e} 1+\mathrm{w} 1$ & $0.65 \pm 0.17$ & $2.88 \pm 0.10$ & 3.4 & 2 \\
Uniform circle disk $\left(0.45^{\circ}\right)$ & $2.35 \pm 0.83$ & $3.29 \pm 0.24$ & 12 & 1 \\
Radio $(4.8 \mathrm{GHz})$ & $1.79 \pm 0.05$ & $2.62 \pm 0.02$ & 0.1 & 0 \\
Radio (4.8 GHz, no ears) & $2.94 \pm 0.16$ & $3.23 \pm 0.04$ & $<0$ & 0 \\
X-ray $(240 \mathrm{PHz})$ & $0.87 \pm 0.01$ & $2.45 \pm 0.03$ & $<0$ & 0 \\
\hline
\end{tabular}

Notes. ${ }^{(a)}$ Model A corresponds to point source model with a power-law spectrum at the best-fit location in Fig. $1 .{ }^{(b)}-2\left(\ln L_{0}-\ln L\right)$ represents the significance of the alternative hypothesis relative to model A (null hypothesis). ${ }^{(c)}$ Additional degrees of freedom. Each uniform disk model is centered at the best-fit position. All the associated uncertainty refers to the $68 \%$ statistic errors. Details on the context are given in Sect. 2.

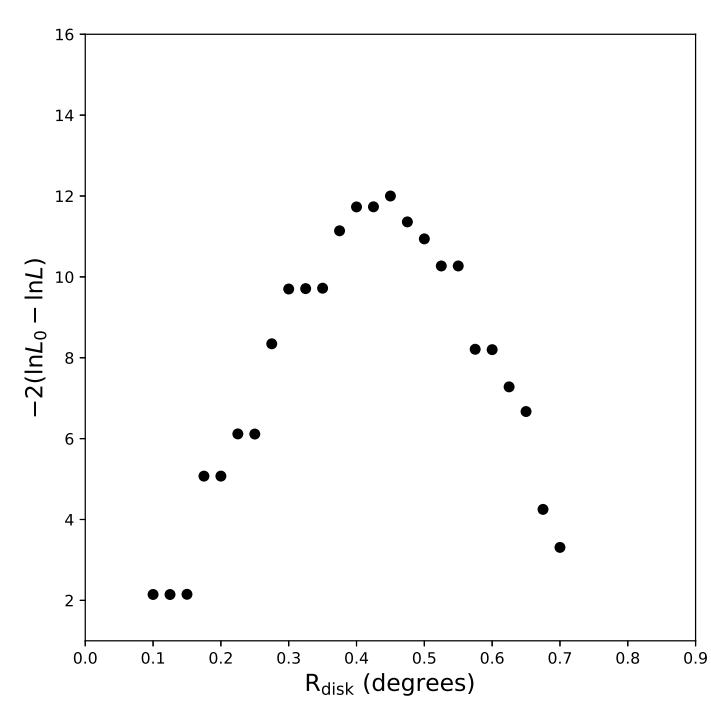

Fig. 2. Significance of the uniform disk models with various radii relative to the single point source model. The maximum likelihood radius of the disk is at $R_{\text {disk }} \sim 0.45^{\circ}$.

statistically. Thus, the GeV emission probably does not originate from the regions of the $\mathrm{TeV}$ emission.

\subsection{Uniform disk tests for spatial extension}

To investigate whether the $\gamma$-ray excess is extended beyond a point source spatially, we assume that the $\gamma$-ray source has uniform surface brightness inside the SNR boundary, and we produced several uniform disk templates centered at the bestfit position with various radii from $0.1^{\circ}$ to $0.7^{\circ}$ in steps of $0.025^{\circ}$. The derived significance of the excess $\gamma$-ray emission is $6 \sigma$ with one additional degree of freedom relative to the single point source. As shown in Fig. 2, the extension likelihood is peaked at the disk radius of $R_{\text {disk }}=0.45^{\circ} \pm 0.06^{\circ}$ ( $1 \sigma$ error), which is roughly compatible with the extension of the radio emission (Geldzahler et al. 1980) longitudinally. The peak significance of $-2\left(\ln L_{0}-\ln L\right)=12(\sim 3.5 \sigma)$ is tentative evidence that the excess $\gamma$-ray emission at the SS433/W50 system is spatially extended. The photon index is $2.62 \pm 0.02$, and $\gamma$-ray luminosity can be estimated as $\sim 6.48 \times 10^{34}(D / 5.5 \mathrm{kpc})^{2} \operatorname{erg~s}^{-1}$.

\subsection{Radio and X-ray templates}

To check if the $\gamma$-ray emission distribution traces the observed radio and $\mathrm{X}$-ray emission, we consider three additional templates. We created two radio emission templates based on the measurements of the Green Bank Observatory at $4.8 \mathrm{GHz}$ with and without two lateral "ears". The X-ray template is produced from the observation with ROSAT Position Sensitive Proportional Counter (ROSAT PSPC) at $1.2 \mathrm{keV}$, taken from the Virtual Observatory SkyView ${ }^{7}$. The contours of the radio and X-ray emission are overlaid in Fig. 1. In Table 1 we list the fitting results for the three templates using the binned likelihood analysis. The significances of the two radio templates relative to the single point source model, both with $\Delta \mathrm{TS}<1$, show no improvement. The significance derived from the case of the X-ray template is significantly lower than the single point source model. This is expected since the excess $\gamma$-ray emission distribution is basically inconsistent with the region of the $\mathrm{X}$-ray emission. Therefore, we reject the hypothesis that the $\gamma$-ray emission originates from the extended region of the X-ray emission.

\subsection{Extension analysis using alternative diffuse background model}

We also used the previous Galactic interstellar emission model gll_iem_v06.fits ${ }^{8}$ to replace the gll_iem_v07.fits in our background model, and repeated the above spatial analyses. In this case, the morphology of the TS map is roughly consistent with the one derived from the latest Galactic interstellar emission model. Under the assumption of a single point-like source model, the derived best-fit position of the excess above $500 \mathrm{MeV}$ is $\left[\mathrm{RA}=287.91^{\circ}, \mathrm{Dec}=5.13^{\circ}\right]$, which is $0.15^{\circ}$ away from the nominal position, and the significance is $\mathrm{TS}=35(6 \sigma)$. A uniform disk template $\left(R_{\text {disk }} \sim 0.45^{\circ}\right)$ can fit the $\gamma$-ray excess with an improvement of $\Delta \mathrm{TS}=24(\sim 5 \sigma)$ relative to the single point-like source model. This shows that inclusion of the latest diffuse background model gll_iem_v07.fits significantly reduces the detection significance, as well as the detected $\gamma$-ray flux. A dedicated analysis of the possible systematic errors due to the diffuse background models is needed.

\footnotetext{
7 https://skyview.gsfc.nasa.gov/current/cgi/titlepage. pl

8 https://fermi.gsfc.nasa.gov/ssc/data/access/lat/ BackgroundModels.html
} 


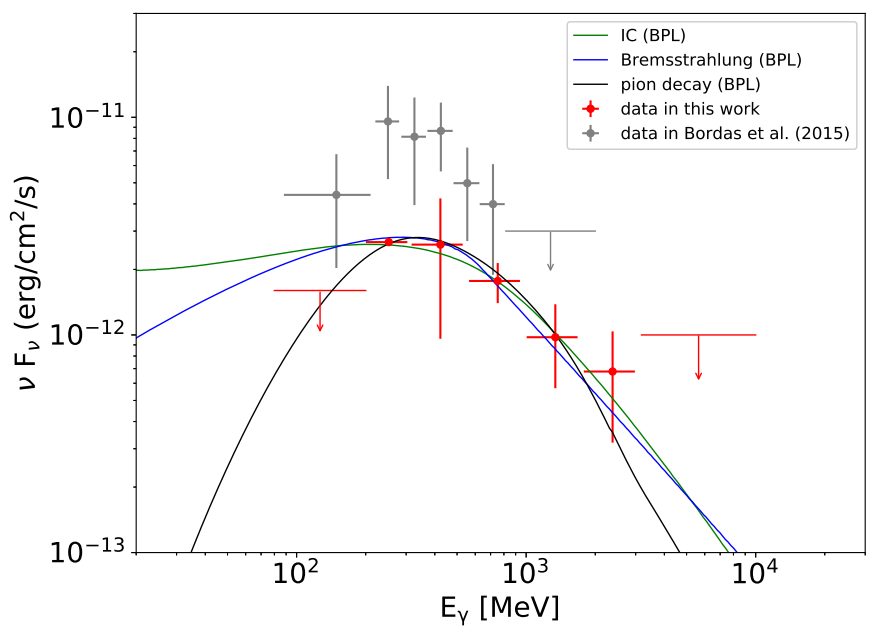

Fig. 3. SED of the GeV emission component of the SS433/W50 system for the $0.45^{\circ}$ uniform circle disk spatial model. The upper limits are calculated within a $3 \sigma$ confidence level. The gray data are taken from Fig. 3 in Bordas et al. (2015).

\section{Spectral analysis}

The results from spatial analysis show that the uniform circle disk template with a radius of $\sim 0.45^{\circ}$ is preferred over the point source for the $\gamma$-ray emission toward the SS433/W50 system, with an improvement of 3.5 to $5 \sigma$. Thus, we fix the $0.45^{\circ}$ uniform circle disk as the spatial model of the extended $\gamma$-ray emission toward the SS433/W50 system to extract the spectral information. The logparabola function, $\frac{\mathrm{d} N}{\mathrm{~d} E} \propto\left(\frac{E}{E_{\mathrm{b}}}\right)^{-\left(\alpha+\beta \log \left(E / E_{\mathrm{b}}\right)\right)}$, can best fit the global spectral shape of the $\gamma$-ray emission with a TS $=39(\sim 6.2 \sigma)$, and the parameters are $\alpha=2.03 \pm 0.01$, $\beta=0.27 \pm 0.02$, and $E_{\mathrm{b}}=0.9 \pm 0.004 \mathrm{GeV}$.

\subsection{Modeling the spectral energy distribution}

To investigate the origin of the extended $\mathrm{GeV}$ emission and the underlying particle spectra that give rise to the observed spectrum of photons, we derived the spectral energy distribution (SED) of SS433/W50 via the maximum likelihood estimation in seven logarithmically spaced energy bins from $80 \mathrm{MeV}-$ $10 \mathrm{GeV}$. For the first and last energy bands, which are detected with a significance of less than $2 \sigma$, we only free the normalization parameters in the iterative model derived from above spatial and spectral fits and calculate the upper limits within $3 \sigma$ confidence level. The energy dispersion correction is applied to the first energy bin. The derived SED of SS433/W50 in this work along with the SED from Bordas et al. (2015) are plotted in Fig. 3. We notice that while the spectral shape is consistent with that in Bordas et al. (2015), the normalization is reduced by a factor of more than 2. This is consistent with a lower detection significance derived in this work (about $5 \sigma$ for point source for ten years data in this work, in comparison with more than $7 \sigma$ for six years data in Bordas et al. 2015). We attribute the difference also to the different choice of the Galactic diffuse background.

We used Naima ${ }^{9}$ (Zabalza 2015) to fit the SED. Naima is a numerical package that includes a set of nonthermal radiative models and a spectral fitting procedure. The code allows us to implement different functions and perform Markov chain

9 http://naima.readthedocs.org/en/latest/index.html\#
Monte Carlo (MCMC; Foreman-Mackey et al. 2013) fitting of nonthermal radiative processes to the data.

To phenomenologically interpret the SED in the GeV band, we adopt both an exponential cut-off power law (ECPL) and a more complex broken power law (BPL) distribution of the radiating electrons or protons. The former is in the form of

$N(E)=A E^{-\alpha} \exp \left[-\left(\frac{E}{E_{\text {cutoff }}}\right)^{\beta}\right]$,

treating $A, \alpha, E_{\text {cutoff }}$, and $\beta$ as free parameters for the fit, and the latter is

$N(E)= \begin{cases}A E^{-\alpha_{1}} & E \leq E_{\text {break }}, \\ A E_{\text {break }}^{\left(\alpha_{2}-\alpha_{1}\right)} E^{-\alpha_{2}} & E>E_{\text {break }},\end{cases}$

with the $A, \alpha_{1}, E_{\text {break }}$, and $\alpha_{2}$ left as free parameters.

In the hadronic scenario, we attribute the observed $\gamma$-rays to the decay of neutral pions produced by the pp collision between the relativistic protons (and nuclei) in the SNR W50 and the protons in the interstellar medium, using the cross-section approximation of Kafexhiu et al. (2014). The average number density of the target protons is assumed to be $4.0 \mathrm{~cm}^{-3}$ as in Bordas et al. (2015). On the other hand, we test the leptonic scenario assuming the $\gamma$-ray emission is produced by the inverseCompton (IC) up-scattering of seed photons, or through the bremsstrahlung of the relativistic electrons embedded in the SNR W50 or matter from the surrounding medium. For the interstellar radiation field of the $\mathrm{IC}$, except for the $\mathrm{CMB}$, we also adopt the infrared and optical emissions from dust and starlight of the local interstellar radiation field calculated by Popescu et al. (2017). For the relativistic bremsstrahlung, we assume the target particle density of $4 \mathrm{~cm}^{-3}$ (Bordas et al. 2015). We adopt the formalism described in Aharonian \& Atoyan (1981) and Khangulyan et al. (2014) for the IC photon spectrum calculation and the relativistic bremsstrahlung spectra from Baring et al. (1999), respectively.

Table 2 lists the best-fit parameters as well as the total energy of the electrons or protons, $W_{\mathrm{e} / \mathrm{p}}$, with $1 \sigma$ statistical errors, searched using the maximum log-likelihood (MML). Figure 3 shows the radiation models with the maximum likelihood values correspondingly. It is evident that the $\pi^{0}$ decay model is the best one to match the flux points, which reveal a pion-bump feature. The parameters are $\alpha_{1}=2.1 \pm 0.2$, $\alpha_{2}=4.0 \pm 0.4, E_{\text {break }}=2.4_{-0.3}^{+0.4} \mathrm{GeV}$, and the total energy of the high-energy protons $W_{\mathrm{p}}=(1.14 \pm 0.05) \times 10^{50} \mathrm{erg}$. The leptonic model of $\mathrm{GeV}$ emission leads to a significant decrease in the maximum likelihood of the fitting, as shown in Table 2. The constraints on leptonic models are mainly from the first two energy bins, which can hardly be addressed by either IC or Bremsstrahlung process except we assume a very sharp low energy cutoff of the low-energy electron spectrum. Such a hadronic origin can also explain the lower significance of the spatial analysis results by using radio templates. The radio emissions are mainly from the synchrotron radiation of electrons and can have a different spatial distribution than that of the proton content, which is responsible for the $\gamma$-ray emission.

\subsection{Upper limits on GeV emission from the e1 region}

Although no $\mathrm{GeV}$ emission is significantly detected at the eastern hotspot region e1 of SS433/W50, the upper limit flux can provide constrains on the leptonic model reported in Abeysekara et al. (2018). We add a point-like source with a power-law 
Table 2. SED fit results for different radiation models.

\begin{tabular}{|c|c|c|c|c|c|c|}
\hline $\begin{array}{l}\text { Radiation } \\
\text { model }\end{array}$ & $\begin{array}{l}\text { Parent particle } \\
\text { distribution }\end{array}$ & $\begin{array}{c}W_{\mathrm{e} / \mathrm{p}}{ }^{(a)} \\
\left(\times 10^{49} \mathrm{erg}\right)\end{array}$ & $\alpha$ or $\alpha_{1}$ & $\begin{array}{c}E_{\text {cutoff }} \text { or } E_{\text {break }} \\
(\mathrm{GeV})\end{array}$ & $\beta$ or $\alpha_{2}$ & $\operatorname{MLL}^{(b)}$ \\
\hline \multirow{3}{*}{$\mathrm{pp}$} & ECPL & $11.9_{-0.3}^{+0.4}$ & $2.1 \pm 0.2$ & $2.7_{-0.3}^{+0.4}$ & 1 (fixed) & -1.6 \\
\hline & ECPL & $12.1_{-0.8}^{+1.1}$ & $2.1_{-0.2}^{+0.3}$ & $2.8_{-0.4}^{+0.6}$ & $1.1 \pm 0.3$ & -1.6 \\
\hline & BPL & $11.4 \pm 0.5$ & $2.1 \pm 0.2$ & $2.4_{-0.3}^{+0.4}$ & $4.0 \pm 0.4$ & -1.0 \\
\hline \multirow{3}{*}{$\mathrm{IC}$} & ECPL & $2.8_{-0.2}^{+0.3}$ & $1.5 \pm 0.1$ & $9.5 \pm 1.2$ & 1 (fixed) & -31.4 \\
\hline & ECPL & $3.7 \pm 0.3$ & $1.7 \pm 0.1$ & $12_{-1.2}^{+1.8}$ & $5.3 \pm 0.7$ & -13.3 \\
\hline & BPL & $3.4_{-0.5}^{+0.3}$ & $1.6 \pm 0.1$ & $8.3_{-0.8}^{+1.4}$ & $6.4_{-0.8}^{+1.4}$ & -10.3 \\
\hline \multirow{3}{*}{ Rel. bremsstrahlung } & ECPL & $1.6 \pm 0.1$ & $1.6 \pm 0.2$ & $1.0_{-0.2}^{+0.4}$ & 1 (fixed) & -10.6 \\
\hline & ECPL & $1.5 \pm 0.1$ & $1.7 \pm 0.2$ & $1.4 \pm 0.3$ & $1.8_{-0.5}^{+0.9}$ & -10.8 \\
\hline & BPL & $1.5 \pm 0.1$ & $1.5 \pm 0.3$ & $0.7_{-0.1}^{+0.3}$ & $3.7_{-0.4}^{+0.7}$ & -7.5 \\
\hline
\end{tabular}

Notes. All errors correspond to a $1 \sigma$ confidence level. ${ }^{(a)}$ Total energy of the electrons or protons. The $W_{\mathrm{p}}$ is calculated based on the protons $>1 \mathrm{GeV}$ and the $W_{\mathrm{e}}$ is calculated based on the electrons $>100 \mathrm{MeV}$. ${ }^{(b)}$ Maximum log-likelihood (MLL).

spectral type at the e1 position into our iterative model, and obtain upper limits within a $2 \sigma$ confidence level. As shown in Fig. 4, we consider the one zone leptonic model as in Abeysekara et al. (2018), namely, the relativistic electrons scatter $\mathrm{CMB}$ photons to $\mathrm{GeV}-\mathrm{TeV}$ energies via an IC process (dot-dashed line) and produce radio to low-energy $\gamma$-ray emissions by the synchrotron process (dashed line). We also assume a power-law electron distribution with an exponential cutoff, that is, $\mathrm{d} N / \mathrm{d} E \propto E^{-\alpha} \exp \left(-E / E_{\max }\right)$. The solid line presents the sum of the IC and synchrotron radiation. We find the first upper limit in the energy range of $300-500 \mathrm{MeV}$ can effectively constrain the $E_{\max }$, and the fitting results are roughly consistent with Abeysekara et al. (2018). The best-fit values of the parameters are $\alpha=2.45_{-0.2}^{+0.1}, E_{\max }=2.5_{-0.3}^{+0.4} \mathrm{PeV}, B=15_{-2}^{+3} \mu \mathrm{G}$, and the total energy of the electrons with energy above $10 \mathrm{GeV}$ is $(1.2 \pm 0.9) \times 10^{47} \mathrm{erg}$. It is interesting that the current bestfit model already predicts a turnover in the energy spectrum in the HAWC energy range. Such kinds of features can be directly tested with more HAWC observations in the future. Furthermore, the next generation $\mathrm{TeV} \gamma$-ray detectors, such as the Cherenkov Telescope Array (CTA; Actis et al. 2011) and the Large High Altitude Air Shower Observatory (LHAASO; LHAASO Collaboration 2016), will definitely provide a clear test on the leptonic model in the termination region of the jet.

\section{Discussion and conclusion}

We have analyzed ten years of Fermi-LAT data toward the SS433/W50 region and found tentative evidence for spatially extended $\mathrm{GeV}$ emission in this region (with a significance of 3.5 to $5 \sigma$, depending on the choice of the diffuse background). The extension of the sub-GeV to $\mathrm{GeV} \gamma$-ray emission are roughly consistent with the size of SNR W50. The SED of these $\gamma$-ray emissions reveals a pion-bump shape and can be well explained by the pion-decay emissions from a population of protons with a low break energy in the spectrum interacting with the ambient gas. Such a slightly accelerated proton population can be injected along the jet or at the jet termination shock (Bordas et al. 2015). The acceleration mechanism should be rather inefficient, given that the $\gamma$-ray spectrum extends only up to a few GeVs. Recently, Rasul et al. (2019) found tentative evidence for periodicity at the

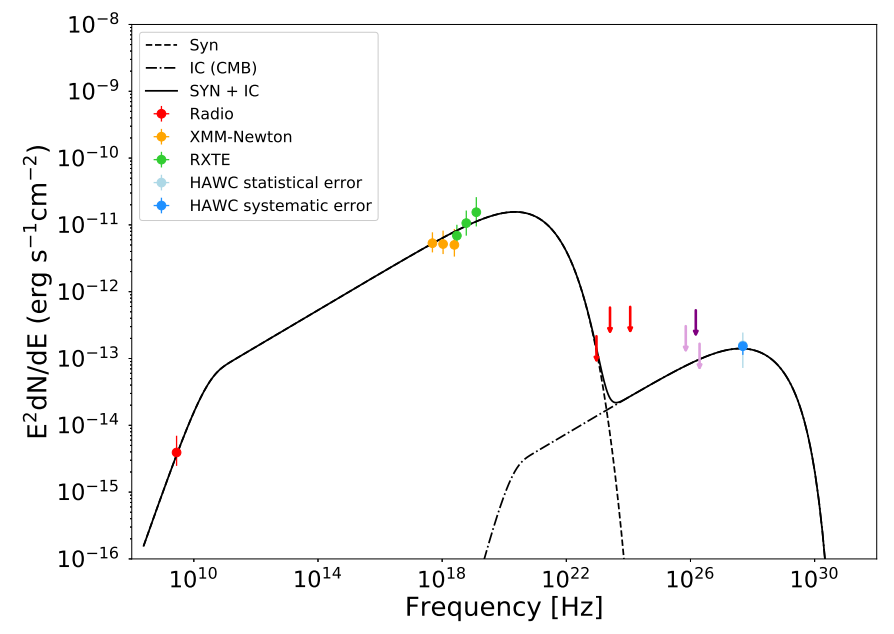

Fig. 4. SED of the eastern emission region e1. The red upper limits are derived in this work within a $2 \sigma$ confidence level. Other data are taken from Abeysekara et al. (2018) directly. Radio to low-energy $\gamma$-ray photons are fitted with a synchrotron emission in a magnetic field (dashed line), and the $\mathrm{GeV}$ to $\mathrm{TeV}$ emission through IC scattering of the CMB (dot-dashed line). The solid line represents the sum of the synchrotron and IC emission.

precession period of the $\mathrm{GeV}$ emissions in SS433. If this is true, at least part of these $\mathrm{GeV}$ emissions may be produced in the basement of the jet, rather than in the termination region or the SNR. However, we note that the significance of the variability is only about $3 \sigma$. We also note that their analysis is performed with old diffuse background models. As mentioned in Sect. 2.5, the update to the latest diffuse background models has significantly reduced the detection significance. A further detailed systematic investigation of diffuse background models in this region is needed to clarify these features. On the other hand, due to the spatial coincidence of the sub-GeV to $\mathrm{GeV} \gamma$-ray emission and the SNR itself, it is more likely that these protons are accelerated in the SNR. We also found that a broken power-law proton spectrum can best fit the $\gamma$-ray SED. Such a broken power-law shape spectrum is also observed in other mid-age SNRs, such as IC433 and W44 (Ackermann et al. 2013). However, the break energy 
here is much lower than that in other mid-age SNRs. Such a lowenergy break may reflect the inefficient acceleration in this SNR due to the presence of the jet. On the other hand, Malkov et al. (2011) have explained the energy break in these mid-age SNRs as being the result of Alfven waves evanescence caused by strong ion-neutral collisions. In this case the break energy is determined by the neutral and ion density, as well as the gas temperatures, which can be very different in W50 due to the influence of the jet.

Due to the different spatial morphology, the $\gamma$-ray emissions observed by Fermi-LAT may have a different origin than that of the $\mathrm{TeV}$ emissions observed by HAWC, which is believed to originate from the termination region of the microquasar jet (Abeysekara et al. 2018). Under this assumption we also extracted the upper limits on the $\mathrm{TeV}$ emission region in Fermi-LAT energy band. We found that the current data can already put interesting constraints on the maximum electron energy in the leptonic model. It is interesting that the current best-fit leptonic model predicts a turnover in the energy spectrum in the HAWC energy range, which can be tested by the further data release of HAWC, as well as the observations of the forthcoming CTA and LHASSO.

Acknowledgements. X.N.S. thanks Yunfeng Liang for helpful discussions. This work is supported by the NSFC under grants 11625312 and 11851304, and the National Key R\&D program of China under the grant 2018 YFA0404203.

\section{References}

Abell, G. O., \& Margon, B. 1979, Nature, 279, 701

Abeysekara, A. U., Albert, A., Alfaro, R., et al. 2018, Nature, 562, 82

Acero, F., Ackermann, M., Ajello, M., et al. 2015, ApJS, 218, 23

Ackermann, M., Ajello, M., Allafort, A., et al. 2013, Science, 339, 807

Actis, M., Agnetta, G., Aharonian, F., et al. 2011, Exp. Astron., 32, 193
Aharonian, F. A., \& Atoyan, A. M. 1981, Ap\&SS, 79, 321

Baring, M. G., Ellison, D. C., Reynolds, S. P., Grenier, I. A., \& Goret, P. 1999, ApJ, 513, 311

Blundell, K. M., \& Bowler, M. G. 2004, ApJ, 616, L159

Bordas, P., Yang, R., Kafexhiu, E., \& Aharonian, F. 2015, ApJ, 807, L8

Brinkmann, W., Pratt, G. W., Rohr, S., Kawai, N., \& Burwitz, V. 2007, A\&A, 463, 611

Dubner, G. M., Holdaway, M., Goss, W. M., \& Mirabel, I. F. 1998, AJ, 116, 1842 Eikenberry, S. S., Cameron, P. B., Fierce, B. W., et al. 2001, ApJ, 561, 1027

Elston, R., \& Baum, S. 1987, AJ, 94, 1633

Fabian, A. C., \& Rees, M. J. 1979, MNRAS, 187, 13P

Farnes, J. S., Gaensler, B. M., Purcell, C., et al. 2017, MNRAS, 467, 4777

Foreman-Mackey, D., Hogg, D. W., Lang, D., \& Goodman, J. 2013, PASP, 125, 306

Geldzahler, B. J., Pauls, T., \& Salter, C. J. 1980, A\&A, 84, 237

Gies, D. R., Huang, W., \& McSwain, M. V. 2002, ApJ, 578, L67

Goodall, P. T., Alouani-Bibi, F., \& Blundell, K. M. 2011a, MNRAS, 414, 2838

Goodall, P. T., Blundell, K. M., \& Bell Burnell, S. J. 2011b, MNRAS, 414, 2828

Kafexhiu, E., Aharonian, F., Taylor, A. M., \& Vila, G. S. 2014, Phys. Rev. D, 90, 123014

Khangulyan, D., Aharonian, F. A., \& Kelner, S. R. 2014, ApJ, 783, 100

Lande, J., Ackermann, M., Allafort, A., et al. 2012, ApJ, 756, 5

LHAASO Collaboration (Di Sciascio, G.) 2016, Nucl. Part. Phys. Proc., 279, 166

Lockman, F. J., Blundell, K. M., \& Goss, W. M. 2007, MNRAS, 381, 881

Malkov, M. A., Diamond, P. H., \& Sagdeev, R. Z. 2011, Nat. Commun., 2, 194

Margon, B. 1984, ARA\&A, 22, 507

Margon, B., Ford, H. C., Grandi, S. A., \& Stone, R. P. S. 1979, ApJ, 233, L63

Milgrom, M. 1979, A\&A, 76, L3

Mirabel, I. F., \& Rodríguez, L. F. 1999, ARA\&A, 37, 409

Popescu, C. C., Yang, R., Tuffs, R. J., et al. 2017, MNRAS, 470, 2539

Rasul, K., Chadwick, P. M., Graham, J. A., \& Brown, A. M. 2019, MNRAS, 485, 2970

Safi-Harb, S., \& Ögelman, H. 1997, ApJ, 483, 868

Safi-Harb, S., \& Petre, R. 1999, ApJ, 512, 784

The Fermi-LAT Collaboration 2019 arXiv e-prints [arXiv: 1902 . 10045]

Xing, Y., Wang, Z., Zhang, X., Chen, Y., \& Jithesh, V. 2019, ApJ, 872, 25

Zabalza, V. 2015, 34th International Cosmic Ray Conference (ICRC2015), 922 